sex, age, sexual orientation, viral transmission, housing, race, education, social occupation and other information on the main comorbidities.

Results There was a predominance of men (73.8\%), white $(57.7 \%)$ and $51 \%$ with incompleted high school. The predominant age groups were 31-40 years (25.6\%), 41-50 (23.8\%) and 51-60 (22.6\%). There was a predominance of heterosexual orientation $(42.3 \%)$, sexual transmission $(81.5 \%)$ and residents in the Urban Zone (81\%). In addition to Occupations: Unemployment (14.9\%), Home (14.3\%) and less favored occupants social positions. The main comorbidities were: Oral Candidiasis (35.1\%), Persistent Dermatitis (23.2\%), Depression (22\%), Pneumonia (14.3\%), Tuberculosis (13.1\%), Syphilis (10.9\%).

Conclusion The profile differs from common sense, calling attention to age and sexual orientation. Finally, there is a need to improve the filling of medical records, for future comparisons and preventions that lead to improvements in care

\section{P189 PREFERENCES OF PEOPLE LIVING WITH HIV FOR INJECTABLE AND ORAL ANTIRETROVIRAL TREATMENT IN THE NETHERLANDS: A DISCRETE CHOICE EXPERIMENT}

${ }^{1}$ I Kremer*, ${ }^{1} \mathrm{C}$ Beaudart, ${ }^{2,3}$ J Simons, ${ }^{4} \mathrm{H}$ Plieger, ${ }^{5} \mathrm{M}$ Schroeder, ${ }^{1} \mathrm{M}$ Hiligsmann. ${ }^{1}$ Maastricht University, Maastricht, The Netherlands; ${ }^{2}$ GSK, Amersfoort, The Netherlands; ${ }^{3}$ University of Groningen, University Medical Centre Groningen, Groningen, The Netherlands; ${ }^{4}$ ViV Healthcare, Amersfoort, The Netherlands; ${ }^{5}$ ViiV HealthCare, London, UK

\subsection{6/sextrans-2021-sti.280}

Background Antiretroviral (ARV) therapies are currently orally administered, daily; however, there are long-acting (LA) injectable alternative ARVs in development. Understanding preferences of people living with HIV (PLWH) for different modes of administration, could provide relevant information for clinical and policy decision-making. This study aimed to elicit preferences of PLWH for LA injectable- compared with oral ARVtherapies, in the Netherlands.

Methods A discrete-choice experiment (DCE) was conducted among PLWH, which presented 12 choice tasks for two ARV therapies (i.e. oral or LA injectable). PLWH were asked in each task to select their preferred ART. Based on literature reviews, interviews with PLWH and expert consultation, ARV options were characterized by six attributes: location of administration, dosing frequency, risk of having short-term side effects, risk of drug-drug interaction, forgivability, and food and mealtime restrictions. Random parameters logit and latent class models were used to estimate preferences of PLWH.

Results A total of 76 PLWH completed the survey. Of the 76 respondents, $22(28.9 \%)$ chose oral ARV therapy in all choice tasks, $30(39.5 \%)$ respondents always chose LA injectable ARV therapy, and $24(31.6 \%)$ respondents varied their choice depending on the choice tasks. Respondents always choosing LA injection reported to be more willing to switch their ARV therapy $(\mathrm{p}=.006)$. On average, PLWH preferred an injectable therapy, a less frequent dosing regimen, lower risks of side effects or drug-drug interactions, therapy administrated at home and therapy without food and mealtime restrictions, compared with alternatives.

Conclusion This study revealed that PLWH have a strong preference for mode of administration. Some people always preferred LA injectable ARV therapy, while others always preferred oral ARVs. This study, therefore, highlights the value and potential marketplace for a LA injectable ARV therapy. Improved medication adherence could result, if patients are actively involved in the decision-making process of their ARV treatment.

\section{P190 DECLINING TRENDS OF SYPHILIS SEROPREVALENCE IN INDIA: A 10-YEAR-ANALYSIS}

P Srivastava*, A Bhargava, S Bansal, N Khunger. Vardhman Mahavir Medical College and Safdarjung Hospital, New Delhi, India, New Delhi, India

\subsection{6/sextrans-2021-sti.281}

Background World Health Organization (WHO) estimates that each year 11 million new cases of syphilis occur globally. Syphilis remains an important public health challenge in lowincome countries like India and has re-emerged in several high-income countries. The present study was undertaken to analyse the trends in prevalence, pattern and clinical presentations of syphilis, over 10 years, at a tertiary-care-hospital in India.

Methods A retrospective observational study was carried out at the Apex Regional STD Centre, Safdarjung Hospital, New Delhi, India; wherein data from patient records of all the syphilis cases from sexually transmitted infections (STIs) clinic, from January 2007 to December 2016 was analysed. Patients were diagnosed using syphilis serological assays as per classical testing strategy. Epidemiological, clinical and investigational data was analysed statistically for changing epidemiological and clinical trends of syphilis.

Results Of 373,845 patients tested for syphilis, $1.41 \%$ males were VDRL reactive compared to $0.49 \%$ females $(\mathrm{x} 2=874.5$, $\mathrm{p}<0.0001)$. VDRL reactivity showed a declining trend with $1.56 \%$ prevalence in 2007 and $1.05 \%$ in 2016 ( $\mathrm{x} 2$ trend $=87.8, \mathrm{p}<0.0001)$ with significant fall in reactivity for females. TPHA reactivity also showed a declining trend $(\mathrm{x} 2$ trend $=32.0, \mathrm{p}<0.0001)$. Biological false positivity was appreciably more in females $(\mathrm{x} 2=4.06, \mathrm{p}=0.043)$ and with VDRL titres $<1: 8(\mathrm{x} 2=44.1, \mathrm{p}<0.0001)$. TPHA positivity was significantly higher with VDRL titres $\geq 1: 8 \quad(\mathrm{x} 2=55.2$, $\mathrm{p}<0.0001)$. Latent syphilis comprised $92.7 \%$ of total diagnosed cases $(p<0.0001)$ and $20-29$ years was the most common age-group with $55.8 \%$ cases $(\mathrm{p}<0.0001)$.

Conclusion Syphilis has features of a disease that could be eliminated; like absence of reservoir, 2-6 week incubation period, availability of inexpensive sero-diagnostic tests and treatable with single dose of penicillin. Since, syphilis has been recommended as a marker of STI prevalence by WHO, the strong and significant decline in seroprevalence of syphilis during 2007-2016, reflects the success of the National STI Prevention and Control Programme whose pillars rest upon education and proper management. 